\title{
THE SENSITIVITY OF THE PASSIVE BIDOMAIN EQUATION TO VARIATIONS IN SIX CONDUCTIVITY VALUES
}

\author{
Barbara M. Johnston and Peter R. Johnston \\ School of Biomolecular and Physical Sciences \\ and \\ Queensland Micro- and Nanotechnology Centre \\ Griffith University, Nathan, QLD, Australia, 4111 email: Barbara.Johnston@griffith.edu.au
}

\begin{abstract}
Previous work has shown that variations in cardiac conductivity values have significant effects on the resulting epicardial potential distributions arising from subendocardial ischaemia. This work carries on from a previous study in this area by allowing for variation in (the more physiologically realistic) six, rather than four, conductivity values. This is achieved by relaxing the usual assumption that the cardiac conductivity values in both normal directions, one normal to the fibres within the sheet and the other normal to the sheet, are equal. An extended non-dimensional formulation is derived for the passive bidomain equation, leading to a normal direction dimensionless conductivity ratio, in addition to the previously found ratios in the transverse and longitudinal directions. Two six-conductivity datasets are used to set up a parameter space for the above ratios, from which epicardial potential distributions are derived and compared. Results from this study appear to support the conclusions of the previous four-conductivity study; that is, differences in the epicardial potential distributions are best explained by variations in the ratio of the intracellular longitudinal conductivity to the intracellular transverse conductivity.
\end{abstract}

\section{KEY WORDS}

Electrocardiography, Bidomain equation, Dimensionless Formulation, Conductivity Values, Mathematical Model, Subendocardial Ischaemia

\section{Introduction}

A number of previous simulation studies [14, 13, 8, 7] have investigated the relationship, between cardiac conductivity values and the epicardial potential distributions, via mathematical models of partial thickness ischaemia applied during the ST segment. Such studies use a bidomain formulation [26], with conductivities in the extracellular $(e)$ and intracellular $(i)$ spaces, in the longitudinal direction $(l)$ along the cardiac fibres, transverse to the fibres $(t)$ within the sheet and normal to the fibres $(n)$, that is, between the sheets. This leads to six conductivity values $g_{e l}, g_{e t}, g_{e n}$, $g_{i l}, g_{i t}$ and $g_{i n}$ and the assumption is usually made that the normal and transverse conductivities are equal, that is, $g_{\text {in }}=g_{i t}$ and $g_{\text {en }}=g_{\text {et }}$.
Experimental studies [5, 4], however, have indicated that this assumption is not valid and a recent simulation study has demonstrated the effect that this assumption has on the form of the resulting epicardial distributions that arise in models of ST segment ischaemia [18]. The aim of this work is to relax this assumption and study the effect variations in conductivity ratios have on the potential distributions.

This work is an extension of previous work by one of the authors [11] that involved the non-dimensionalised passive bidomain equation and three dimensionless conductivity ratios. Experimentally derived four-conductivity datasets $[1,21,22]$ were used to set up a parameter space for these ratios, from which epicardial potential distributions were derived [12]. These distributions were then compared using correlation coefficients. That work assumed that the conductivity values in the normal direction were the same as those in the transverse direction. This new study removes that restriction and extends the nondimensional formulation to include two normal direction conductivities, which leads to an additional dimensionless conductivity ratio. Based on this work, a new sensitivity study is carried out, again involving a parameter space of the conductivity ratios and comparisons between epicardial potential distributions. This study uses the only six conductivity datasets that are available, those of MacLachlan et al. [17], which drew on the study of Foster and Schwan [2], and Hooks et al. [4].

This paper begins with a description of the governing equations, geometry and boundary conditions for the model. Then the passive bidomain equation is nondimensionalised and from this conductivity ratios are derived. These are used to find a parameter space to use in the sensitivity study, which aims to explain variations in the potential distributions which result from modelling partial thickness ischaemia during the ST segment. Results for the study are then presented and discussed, leading to overall conclusions. 


\section{Methods}

\subsection{Governing Equations and Model Geometry}

During the ST segment of the electrocardiogram, the electric potential can be modelled in a region of cardiac tissue by the passive bidomain equation [7]

$$
\nabla \cdot\left(\mathbf{M}_{i}+\mathbf{M}_{e}\right) \nabla \phi_{e}=-\nabla \cdot \mathbf{M}_{i} \nabla \phi_{m}
$$

where $\phi_{e}$ is the extracellular potential in the cardiac tissue, $\phi_{m}$ is the given transmembrane potential distribution and $\mathbf{M}_{e}$ and $\mathbf{M}_{i}$ are conductivity tensors for the extracellular $(e)$ and intracellular $(i)$ spaces, respectively.

The conductivity tensors are of the form [3]

$$
\mathbf{M}_{h}=\mathbf{A G}_{h} \mathbf{A}^{T}
$$

where $h=e$ or $i$ and $\mathbf{G}_{h}$ is a diagonal matrix of conductivity values ( $g_{h l}$, along the fibre direction (longitudinal), $g_{h t}$, across the sheets of fibres (transverse) and $g_{h n}$, between the sheets of fibres (normal)), and the matrix $\mathbf{A}=\left\{a_{i j}\right\}$ is a rotation matrix which maps the local direction of the fibres to the global coordinate system. Given these assumptions, then $\mathbf{M}_{h}$ can be shown to have the form

$$
\mathbf{M}_{h}=g_{h t} \mathbf{I}+\left(g_{h n}-g_{h t}\right) \mathbf{K}+\left(g_{h l}-g_{h t}\right) \hat{\mathbf{a}}^{T} \hat{\mathbf{a}}
$$

where $\mathbf{I}$ is the $3 \times 3$ identity matrix, $\mathbf{K}$ is the $3 \times 3$ zero matrix, apart from $k_{33}=1$, and $\hat{\mathbf{a}}$ is a unit vector which indicates the local fibre direction [20].

The blood mass and cardiac tissue are assumed to be in contact and the behaviour of the electric potential in the blood mass, $\phi_{b}$, is governed by Laplace's equation

$$
\nabla^{2} \phi_{b}=0 .
$$

In this study, the computational domain is represented by a block of cardiac tissue of finite size in the $x$ and $y$ directions and of unit thickness in the $z$-direction. The origin of the rectangular $(x, y, z)$ coordinate system is situated in the centre of the block of tissue, with the epicardial surface represented by the $x-y$ plane $(z=0)$ and the endocardial surface at $z=1$. It is assumed that the endocardial surface is in contact with a finite-sized blood mass for $z>1$. An ischaemic region is placed at the centre of the block of tissue and this extends part way from the endocardium towards the epicardium (Figure 1).

The transition from normal to ischaemic tissue, during the ST segment, is described by a smooth variation in the transmembrane potential, via exponential and hyperbolic functions $[26,14]$. The plateau potential difference (between normal tissue and ischaemic tissue), $\Delta \phi_{p}$, is set to $-30 \mathrm{mV}$. Here it is assumed, as per previous studies $[14,13]$, that fibre rotation is through an angle of $120^{\circ}$ from the epicardium to the endocardium (Figure 1). The fibres on the epicardium are aligned with the positive $x$-axis, and so the longitudinal direction at any depth $z$, with respect to the direction of the positive $x$-axis, is given by $g(z)=\frac{2 \pi}{3} z$.

\begin{tabular}{ll|ll}
\hline Tissue & & & $z=0$ \\
\hline Blood & & & \\
& & & \\
& & & \\
& &
\end{tabular}

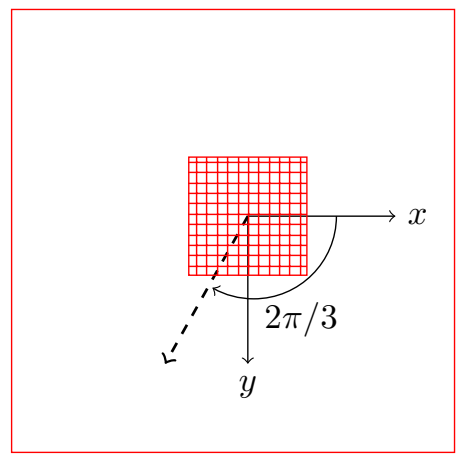

Figure 1. Two views of the tissue-blood model used in this study. The upper panel is a cross-sectional view of the tissue $(z \in[0,1])$ and the blood (for $z>1$ ). The epicardium is at $z=0$ and the endocardium is at $z=1$. The checked region indicates ischaemic tissue. The lower panel is a plan view of the model, looking from the negative $z$ direction, again with the checked region indicating the ischaemic tissue. The dashed arrow indicates the fibre direction on the endocardium when the direction of the fibres on the epicardium is along the $x$-axis.

To take account of the rotation in the conductivity tensor, it is also assumed that

$$
\mathbf{A}=\left(\begin{array}{ccc}
\cos g(z) & \sin g(z) & 0 \\
-\sin g(z) & \cos g(z) & 0 \\
0 & 0 & 1
\end{array}\right)
$$

\subsection{Boundary Conditions}

It is assumed that the epicardial surface at $z=0$ is insulated, i.e.

$$
\text { at } z=0, \quad \frac{\partial \phi_{e}}{\partial z}=0
$$

This is chosen to be consistent with earlier simulations $[14,13]$ and to match experimental work, where measurements of epicardial potentials were made in an open chest experiment [16]. It is also assumed that the edges of the tissue and the blood mass in the $x$ and $y$ directions are insulated, i.e.

$$
\begin{gathered}
\mathbf{M}_{e} \nabla \phi_{e} \cdot \mathbf{n}=0 \text { for } 0 \leq z \leq 1 \\
\nabla \phi_{b} \cdot \mathbf{n}=0 \text { for } z \geq 1
\end{gathered}
$$

where $\mathbf{n}$ is the outward normal. At the bottom of the blood, $\phi_{b}=0$. Finally, potential and current are assumed continuous between the tissue and the blood mass, so at $z=1$

$$
\phi_{e}=\phi_{b} \quad \text { and } \quad g_{e n} \frac{\partial \phi_{e}}{\partial z}=g_{b} \frac{\partial \phi_{b}}{\partial z}
$$


where $g_{b}$ is the blood conductivity. These boundary conditions are consistent with previous studies [14, 13, 8], where it was concluded that, taking the scale of the problem into consideration, the boundary conditions are justified [23]. Although the boundary conditions have recently been reconsidered and modified [19], the changes have been shown to have only a small effect when ST segment shifts are modelled [9].

\subsection{Non-dimensional Formulation}

The governing equations will be non-dimensionalised as follows, where $\Delta \phi_{p}$ is the difference between transmembrane potentials in normal tissue and ischaemic tissue: let $\Phi_{m}=\phi_{m} / \Delta \phi_{p}, \Phi_{e}=\phi_{e} / \Delta \phi_{p}$ and $\Phi_{b}=\phi_{b} / \Delta \phi_{p}$ and the dimensionless tensors

$$
\mathbf{N}_{i}=\frac{\mathbf{M}_{i}}{g_{i t}+g_{e t}} \text { and } \mathbf{N}_{e}=\frac{\mathbf{M}_{e}}{g_{i t}+g_{e t}} .
$$

Then the governing equations (1) and (4) are

$$
\nabla \cdot\left(\mathbf{N}_{i}+\mathbf{N}_{e}\right) \nabla \Phi_{e}=-\nabla \cdot \mathbf{N}_{i} \nabla \Phi_{m}
$$

and

$$
\nabla^{2} \Phi_{b}=0
$$

with suitable adjustments to the boundary conditions. The interface conditions for the tissue and blood are then

$$
\Phi_{e}=\Phi_{b} \text { and } \frac{g_{e n}}{g_{i t}+g_{e t}} \frac{\partial \Phi_{e}}{\partial z}=\frac{g_{b}}{g_{i t}+g_{e t}} \frac{\partial \Phi_{b}}{\partial z} .
$$

Now, in the notation of Potse et al. [20],

$$
\mathbf{N}_{i}+\mathbf{N}_{e}=\frac{\mathbf{M}_{i}+\mathbf{M}_{e}}{g_{i t}+g_{e t}}=\mathbf{I}+(\gamma-1) \mathbf{K}+(\delta-1) \hat{\mathbf{a}}^{T} \hat{\mathbf{a}}
$$

where $\mathrm{a}$ is a unit vector which describes the fibre direction and

$$
\delta=\frac{g_{i l}+g_{e l}}{g_{i t}+g_{e t}}, \gamma=\frac{g_{i n}+g_{e n}}{g_{i t}+g_{e t}}
$$

This new quantity $\gamma$ is additional to $\delta$, which was found as part of the previous analysis [12].

\subsection{Conductivity Ratios}

The conductivity tensor $\mathbf{N}_{i}$ is now of the form

$$
\mathbf{N}_{i}=\frac{g_{i t}}{g_{i t}+g_{e t}} \mathbf{I}+\frac{g_{i n}-g_{i t}}{g_{i t}+g_{e t}} \mathbf{K}+\frac{g_{i l}-g_{i t}}{g_{i t}+g_{e t}} \hat{\mathbf{a}}^{T} \hat{\mathbf{a}}
$$

which contains three dimensionless groups

$$
\frac{g_{i t}}{g_{i t}+g_{e t}}=\frac{1}{1+\frac{g_{e t}}{g_{i t}}}, \frac{g_{i n}-g_{i t}}{g_{i t}+g_{e t}}=\frac{\frac{g_{i n}}{g_{i t}}-1}{1+\frac{g_{e t}}{g_{i t}}}
$$

and

$$
\frac{g_{i l}-g_{i t}}{g_{i t}+g_{e t}}=\frac{\frac{g_{i l}}{g_{i t}}-1}{1+\frac{g_{e t}}{g_{i t}}}
$$

\begin{tabular}{ccc}
\hline Quantity & MacLachlan et al. [17] & Hooks et al. [4] \\
\hline$g_{i l}$ & 3.0 & 2.63 \\
$g_{i t}$ & 1.0 & 0.263 \\
$g_{i n}$ & 0.31525 & 0.08 \\
$g_{\text {el }}$ & 2.0 & 2.63 \\
$g_{\text {et }}$ & 1.65 & 2.45 \\
$g_{\text {en }}$ & 1.3514 & 1.087 \\
\hline$g_{i l} / g_{i t}$ & 3.0 & 10.0 \\
$g_{i n} / g_{i t}$ & 0.32 & 0.31 \\
$g_{\text {et }} / g_{i t}$ & 1.7 & 9.3 \\
$g_{b} / g_{i t}$ & 2.4 & 25.5 \\
\hline$\delta$ & 1.89 & 1.94 \\
$\gamma$ & 0.63 & 0.43 \\
\hline
\end{tabular}

Table 1. Conductivity data and ratios for the indicated experimental studies (in $\mathrm{mS} / \mathrm{cm}$ ), as well as the quantities given in Equation (15) used in this work.

leading to three naturally occurring conductivity ratios,

$$
\frac{g_{i l}}{g_{i t}} \text { and } \frac{g_{e t}}{g_{i t}} \text { and } \frac{g_{e n}}{g_{i t}}
$$

the first two of which occurred in the previous work [11, 12].

The right hand side of boundary condition (13) also leads to a fourth conductivity ratio $\frac{g_{b}}{g_{i t}}$, i.e.

$$
\frac{g_{b}}{g_{i t}+g_{e t}}=\frac{\frac{g_{b}}{g_{i t}}}{1+\frac{g_{e t}}{g_{i t}}}
$$

The ratios $g_{i l} / g_{i t}, g_{i n} / g_{i t}, g_{e t} / g_{i t}$ and $g_{b} / g_{i t}$ are given, for the conductivity data sets of MacLachlan et al. [17] and Hooks et al. [4], in Table 1, along with values for $\delta$ and $\gamma$. The blood conductivity $g_{b}$ is set to $6.7 \mathrm{mS} / \mathrm{cm}$ [24] for the data of Hooks et al. [4], since no value is given therein. Note that the conductivities $g_{e l}$ and $g_{e n}$ are only expressed implicitly in this formulation of the bidomain equation.

Previous work $[11,12]$ has found that $\delta$ (the ratio of total longitudinal conductivity to total transverse conductivity) is approximately 3.2 for the experimentally obtained four-conductivity datasets. But, using the six conductivity data (Table 1), this ratio is approximately 1.9 for both datasets and this is the value that will be used in this study. Values calculated for $\gamma$ in Table 1 are 0.63 and 0.43 . Now consider the ratios of total conductivities, total longitudinal conductivity:total transverse conductivity: total normal conductivity, giving

$$
g_{i l}+g_{e l}: g_{i t}+g_{e t}: g_{i n}+g_{e n}=\delta: 1: \gamma
$$

Taking $\delta=1.9$ and using the experimental result of Hooks et al. [6] that indicates that these ratios should be approximately $4: 2: 1$, leads to the suggestion of taking $\gamma=0.5$. This lies between the two values in Table 1 and leads to 
a ratio of 3.8:2:1. The other consistent ratio in Table 1 is the value for $g_{i n} / g_{i t}$, which is 0.32 and 0.31 . This ratio was identified as one of the naturally occurring ratios in the above analysis, but, in the absence of other information it will be left fixed at 0.3 in this work.

If these values are adopted, the choice of conductivity data set does not affect the left hand side of equation (11), with the consequence that all differences in conductivity data sets arise from the right hand side of equation (11). For this study $\delta$ will be taken to be 1.9 and $\gamma$ will be set at 0.5 , along with $g_{i n} / g_{i t}=0.3$.

\subsection{Study Parameters}

To be consistent with the previous study [12] and to take account of the conductivity values in Table 1, the parameter space for the sensitivity study is chosen to be:

1. Set $g_{i l} / g_{i t}$ to be $3,5,7,9,11$.

2. Set $g_{e t} / g_{i t}$ to be $1,5,9,13$.

3. Set $g_{b} / g_{i t}$ to be $5,10,15,25,35$.

4. The values of $g_{e l} / g_{i t}$ and $g_{e n} / g_{i t}$ are calculated, using (15), as follows (with $\delta=1.9, \gamma=0.5$ and $g_{i n} / g_{i t}=$ $0.3)$

$$
\frac{g_{e l}}{g_{i t}}=\delta\left(1+\frac{g_{e t}}{g_{i t}}\right)-\frac{g_{i l}}{g_{i t}} .
$$

and

$$
\frac{g_{e n}}{g_{i t}}=\gamma\left(1+\frac{g_{e t}}{g_{i t}}\right)-\frac{g_{i n}}{g_{i t}} .
$$

There are a number of things that can be observed from the above parameter space. Firstly, in nearly all cases the value of the ratio $g_{i n} / g_{i t}$ in (21) has a negligible effect on $g_{e n} / g_{i t}$, providing a justification for leaving it fixed at 0.3. However, this means that, unlike the value of $g_{e l} / g_{i t}$, which is affected by changes in $g_{i l} / g_{i t}$ and $g_{e t} / g_{i t}$, the ratio $g_{\text {en }} / g_{i t}$ is only affected by changes in $g_{\text {et }} / g_{i t}$. Another point to note is that the data of MacLachlan et al. [17] sit on a corner of the parameter space, unlike the set of Hooks et al. [4] which lies quite close to the centre of the parameter space. Lastly, when $g_{e t} / g_{i t}=1$, all values of $g_{i l} / g_{i t}>$ 3 lead to negative values of $g_{e l} / g_{i t}$, and so these values were removed from the study since they are physiologically unrealistic.

The finite volume method [10] was used to solve equations (11) and (12) and then the epicardial potential distributions were compared visually and via the correlation coefficient

$$
C C=\frac{\sum_{i=1}^{n}\left[\left(\phi_{1}\right)_{i}-\overline{\phi_{1}}\right]\left[\left(\phi_{2}\right)_{i}-\overline{\phi_{2}}\right]}{\left\|\phi_{1}-\overline{\phi_{1}}\right\|\left\|\phi_{2}-\overline{\phi_{2}}\right\|}
$$

where $\phi_{1}$ and $\phi_{2}$ are vectors which represent the two epicardial potential distributions and $\overline{\phi_{j}}, j=1,2$, is the average value of each of these. The correlation coefficient is an appropriate method of comparison between two potential distributions as it is often used in this way in electrocardiography [15].

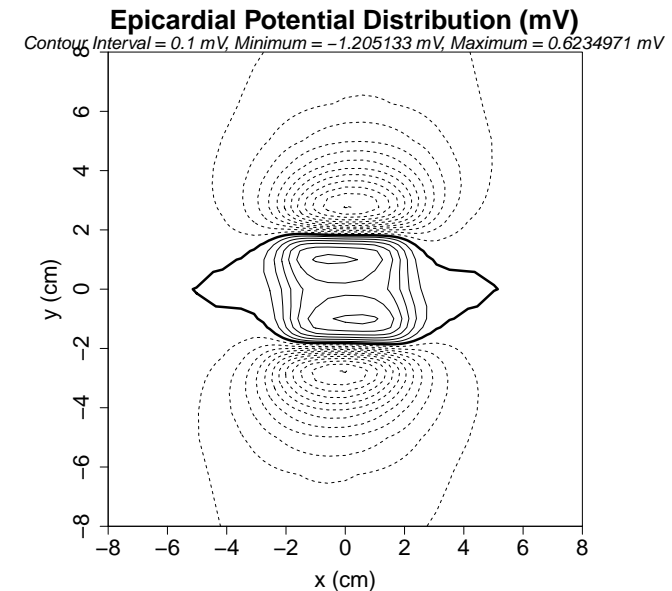

(a) Hooks et al. [4]

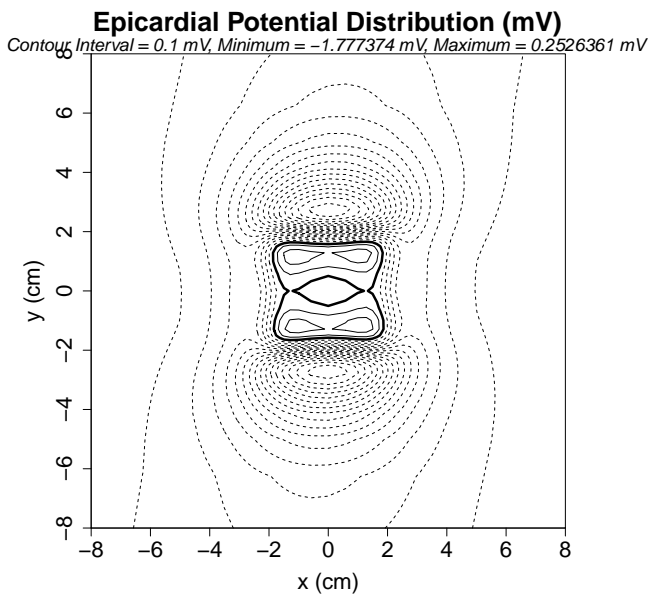

(b) MacLachlan et al. [17] with $g_{b}=2.39$

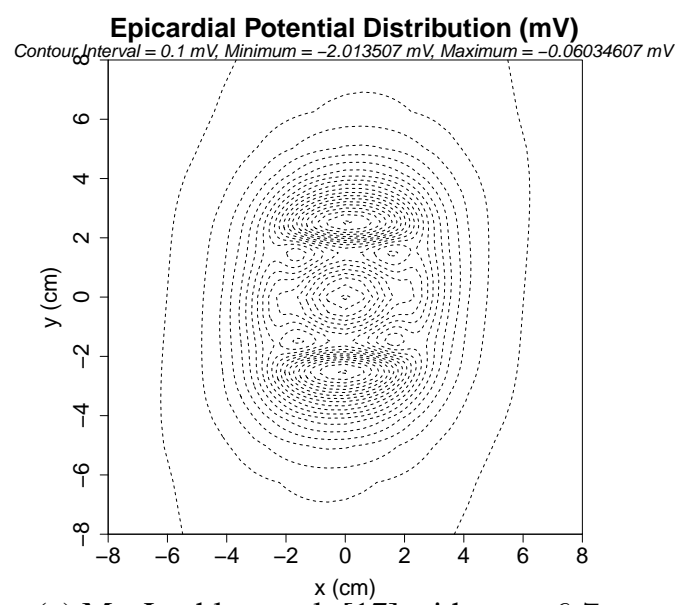

(c) MacLachlan et al. [17] with $g_{b}=6.7$

Figure 2. Dimensionless epicardial potential distributions in a block model of cardiac tissue for two different conductivity data sets. The plot in (a) is generated from the data of Hooks et al. [4] and plots in (b) and (c) come from the data of MacLachlan et al. [17] with $g_{b}=2.39$ and $g_{b}=6.7$ $\mathrm{mS} / \mathrm{cm}$ respectively. In all figures, solid lines indicate positive potentials, dashed lines indicate negative potentials and the zero of potential is indicated by the thicker solid line. A contour interval of 0.1 is used throughout. 


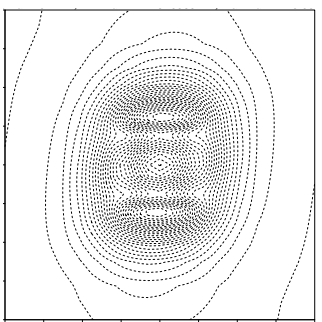

10

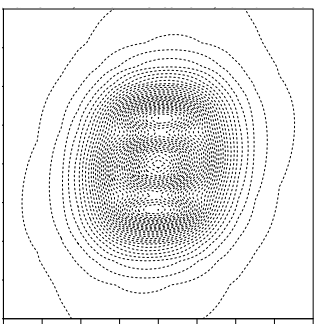

$g_{b} / g_{i t}$

15

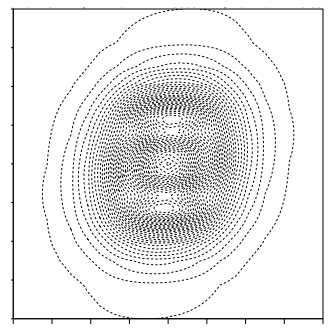

25

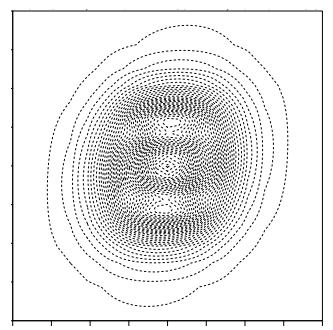

35

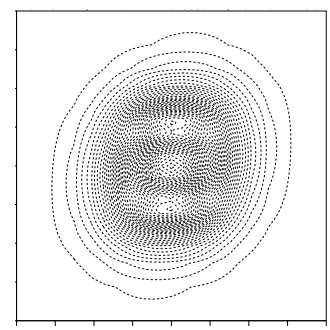

Figure 3. Epicardial potential distributions (dimensionless) in a block model of cardiac tissue with different blood conductivity ratios $g_{b} / g_{i t}$, where $g_{i l} / g_{i t}=3$ and $g_{e t} / g_{i t}=1$. A contour interval of 0.1 is used throughout.

\section{Results}

In this study, a block of cardiac tissue of thickness $1 \mathrm{~cm}$ and size $16 \mathrm{~cm} \times 16 \mathrm{~cm}$ in the $x$ and $y$ directions [14], was used. A solution for the governing equations was found using the finite volume method [10], with a blood mass depth of $26 \mathrm{~cm}$ whose bottom face was chosen to be the zero of potential. The ischaemic region was set to be $4 \mathrm{~cm} \times$ $4 \mathrm{~cm}$ in the $x$ and $y$ directions, and was centrally positioned and covered $50 \%$ of the depth between the endocardium and the epicardium. The ischaemic borders were set to be 0.01 , which led to a steep interface between normal and ischaemic tissue. As mentioned above, fibre rotation was set at $120^{\circ}$ [25].

Figure 2 shows the epicardial potential distributions, obtained for the conductivity data sets of (a) Hooks et al. [4] and (b) and (c) MacLachlan et al. [17]. The distribution in (b) is calculated using the value $g_{b}=2.39 \mathrm{mS} / \mathrm{cm}$ mentioned in their study, whereas (c) is calculated using $g_{b}=6.7 \mathrm{mS} / \mathrm{cm}$ consistent with that used for (a) and previous work $[11,12]$. The conductivity data set of Hooks et al. [4] in (a) leads to a region of positive potential gradients above the region of ischaemia, similar to (b) and in contrast to (c) where there are three potential wells. The objective of this study is to investigate these differences.

As discussed previously, the only plots that make physiological sense for $g_{e t} / g_{i t}=1$ are those where $g_{i l} / g_{i t}=3$. A set of these is presented in Figure 3 for a range of values of $g_{b} / g_{i t}$. The various plots in this figure are very similar, giving correlations, for $g_{b} / g_{i t}=$ $10,15,25,35$ with $g_{b} / g_{i t}=5$, of $0.997,0.995,0.993$ and 0.992 , respectively.

Figure 4 shows a set of epicardial potential distributions where the ratio $g_{b} / g_{i t}$ is set to 10 . The ratio $g_{e t} / g_{i t}$ increases across the rows and the ratio $g_{i l} / g_{i t}$ increases down the columns. The numbers under each figure represent a pair of correlation coefficients (equation (22)), where the first in each pair is the correlation coefficient of that distribution relative to the final distribution in that row $\left(g_{e t} / g_{i t}=13\right)$ and the second of the pair is the correlation coefficient of that distribution compared with the final distribution in that column $\left(g_{i l} / g_{i t}=11\right)$. Figure 5 is presented in the same way, except that in this case $g_{b} / g_{i t}=25$. Similar figures (not presented) were prepared for each of the $g_{b} / g_{i t}$ ratios.

Study of Figures 4 and 5 and similar figures shows that variations in any of the conductivity ratios $g_{i l} / g_{i t}$, $g_{e t} / g_{i t}$ and $g_{b} / g_{i t}$ can result in substantial changes in the generated epicardial potential distributions. Within a figure (ie. where $g_{b} / g_{i t}$ is constant), the pattern across the rows from the top left to the bottom right generally seems to involve a change from three potential wells to a central region of positive potential, surrounded by two regions of negative potential. The main effect associated with an increase in the ratio $g_{b} / g_{i t}$ (ie. from one figure to the next) seems to be a delay in the onset of this change. The fact that the second in each pair of correlation coefficients (ie. the column correlations) is lower as $g_{b}$ increases is also consistent with this observation.

Also, within a figure, correlations increase across a row (ie. as $g_{e t} / g_{i t}$ increases while $g_{i l} / g_{i t}$ is held constant) and similarly, when $g_{e t} / g_{i t}$ is held constant and $g_{i l} / g_{i t}$ increases down a column, correlations again increase, in almost every case. This shows that changes in either of these ratios affects the form of the epicardial potential distribution. A study of the correlation coefficients across rows and down columns indicates, however, that changes in the ratio $g_{e t} / g_{i t}$ are less significant than changes in the ratio $g_{i l} / g_{i t}$, a result that was also found in the previous study [12] using the four-conductivity datasets.

Another interesting observation for any one of the figures is that, in addition to correlations increasing across a row, they also continue to increase, in most cases, from the end of one row to the start of the next row (with some exceptions in the top row).

Other points to note are that the data for the plots in Figure 3 sit on the corner of the parameter space and are qualitatively very different from all other distributions, ex- 


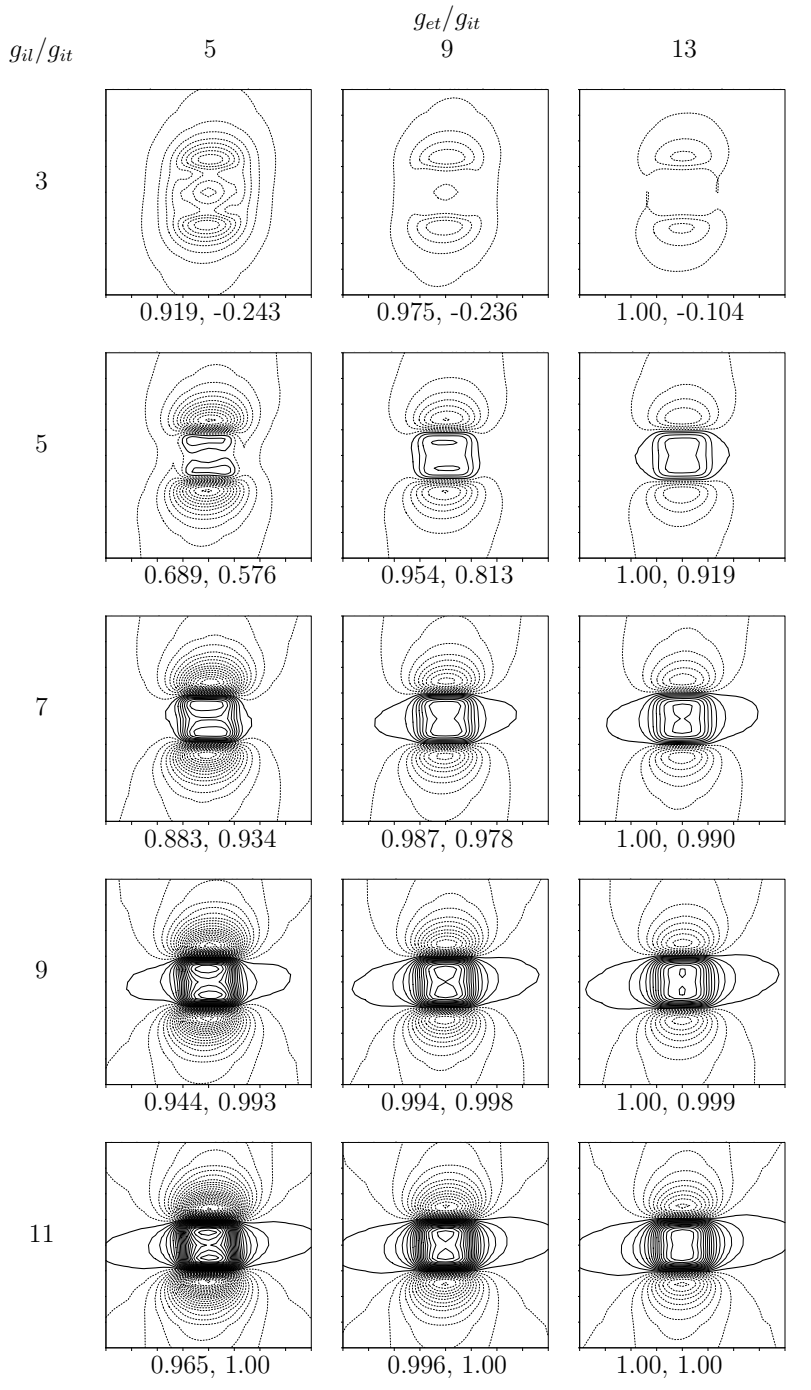

Figure 4. Epicardial potential distributions (dimensionless) in a block model of cardiac tissue with different conductivity ratios $g_{i l} / g_{i t}$ and $g_{e t} / g_{i t}$ for a fixed ratio of $g_{b} / g_{i t}=10$. The value of $g_{e t} / g_{i t}$ is 5,9 and 13, respectively, across the columns and the value of $g_{i l} / g_{i t}$ is 3,5, 7, 9 and 11, respectively, down the rows. A contour interval of 0.1 is used throughout. The numbers below each figure represent correlation coefficients, for the rows and columns, respectively, as explained in the text.

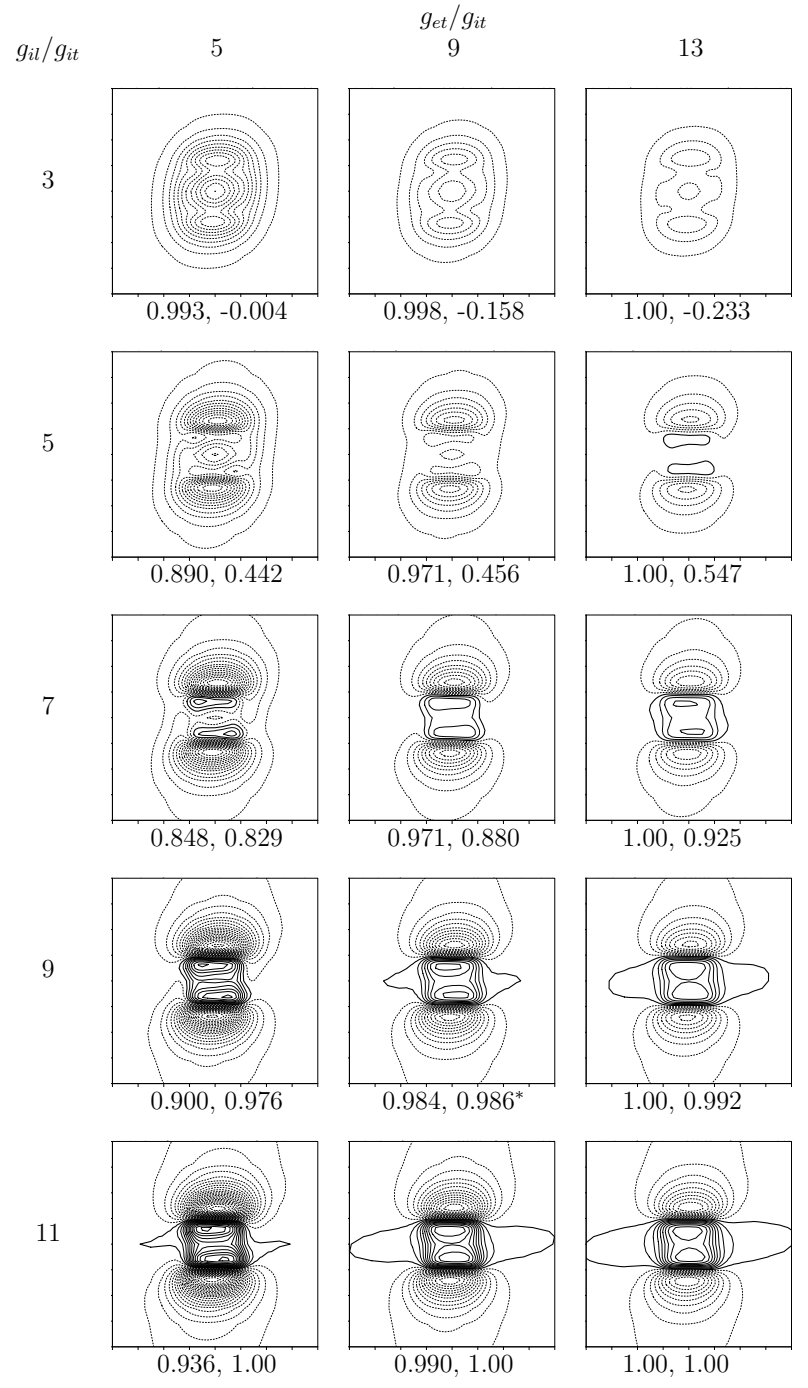

Figure 5. Epicardial potential distributions (dimensionless) in a block model of cardiac tissue with different conductivity ratios $g_{i l} / g_{i t}$ and $g_{e t} / g_{i t}$ for a fixed ratio of $g_{b} / g_{i t}=25$. The value of $g_{e t} / g_{i t}$ is 5,9 and 13, respectively, across the columns and the value of $g_{i l} / g_{i t}$ is $3,5,7,9$ and 11 , respectively, down the rows. The format in this figure is the same as for Figure 4. The panel marked with a ${ }^{*}$ approximates the distribution from the data of Hooks et al. [4] in Figure 2(a). 
cept some of those where $g_{i l} / g_{i t}=3$ (Figures 4 and 5). When $g_{b} / g_{i t}=5$ (and $g_{e t} / g_{i t}=1, g_{i l} / g_{i t}=3$ ), the data correspond approximately to those of MacLachlan et al. [17] (Table 1) and the plot is similar to Figure 2(c), rather than (b), which is not surprising since $g_{b} / g_{i t}=5$ is closer to 6.7 in (c) than 2.39 in (b). The plot that is generated from data that is closest to that of Hooks et al. [4] in Figure 2(a) is the one in Figure 5 where both $g_{i l} / g_{i t}$ and $g_{\text {et }} / g_{i t}=9$ and these two plots are very similar, including in terms of values of potential.

\section{Discussion}

In both cases, the datasets of Hooks et al. [4] and MacLachlan et al. [17] generate epicardial potential distributions (Figures 2(a) and (b), respectively), which reflect key features of experimental studies in sheep [16]; that is, firstly, that, near the ischaemic boundaries, there are large potential gradients and secondly that ST elevation is found above the ischaemic region. However, if the generally accepted value for the conductivity of blood $g_{b}$ of $6.7 \mathrm{mS} / \mathrm{cm}$ is used (Figure 2(c)) rather than the MacLachlan et al. [17] study value of $2.39 \mathrm{mS} / \mathrm{cm}$, the potential distribution now has three potential wells, with no ST elevation.

An analysis of the data of MacLachlan et al. [17] (Table 1) shows that it lies near a corner of the parameter space and that other potential distributions in this region of parameter space (ie. where $g_{i l} / g_{i t}=3$ ) also do not show any ST elevation (Figures 3-4). On the other hand, the data of Hooks et al. [4], lie towards the centre of the parameter space and the resulting potential distribution (Figure 5) is reasonably representative of the distributions obtained for many of the parameter combinations. The general trend, as either $g_{e t} / g_{i t}$ or $g_{i l} / g_{i t}$ increases, is for ST elevation to develop above the ischaemic region, with the first appearance of elevation occurring earlier for lower values of $g_{b} / g_{i t}$.

An interesting feature of each set of potential distributions corresponding to a fixed $g_{b} / g_{i t}$ ratio (for example, Figures 4 and 5), relates to the first value of the correlation coefficient in each pair (ie. correlations with the final column where $g_{e t} / g_{i t}=13$ ). These correlations not only increase across each row, but they continue to increase to the start of the following row and then across it, and so on (with a few exceptions in the top row). This result seems to be consistent with the conclusion (which can be verified by studying the correlation values across the rows and down the columns) that changes in the $g_{i l} / g_{i t}$ ratio affect the resulting epicardial potential distribution more significantly than changes in $g_{e t} / g_{i t}$.

\section{Conclusion}

This study has involved the extension of a formulation of the passive bidomain equation, obtained by nondimensionalisation, to include six independently varying conductivity values, rather than making the usual assump- tion that the normal and transverse conductivities are equal. This formulation was then used to define a parameter space associated with the conductivity ratios that arose from the formulation, based on the two available six-conductivity datasets. These parameters were used in a sensitivity study, to try to find reasons for differences in the epicardial potential distributions that arise when different sets of conductivity values are used to model partial thickness ischaemia during the ST segment.

Results from the study show that, when the assumption is made to fix the ratio $g_{i n} / g_{i t}$ (based on the values in the conductivity datasets of Hooks et al. [4] and MacLachlan et al. [17]), and a fixed ratio of $g_{b} / g_{i t}$ is considered, more significant differences in the epicardial potential distribution can be seen when the ratio of $g_{\text {et }} / g_{i t}$ is fixed and the ratio $g_{i l} / g_{i t}$ is varied than vice versa. This leads to the same conclusion as the previous four-conductivity study [12], namely that the ratio $g_{i l} / g_{i t}$ (ie. the intracellular conductivity along the fibres) is the key to significant changes in the epicardial potential distribution, rather than the ratio $g_{\text {et }} / g_{i t}$ (ie. the extracellular conductivity transverse to the fibres but within the sheet).

\section{References}

\section{References}

[1] L. Clerc, Directional differences of impulse spread in trabecular muscle from mammalian heart, Journal of Physiology, 255, 1976, 335-346.

[2] K. R. Foster and H. P. Schwan, Dielectic properties of tissue and biological materials: A critical review, Critical Reviews in Biomedical Engineering, 17(1), 1989, 25-104.

[3] R. M. Gulrajani, Bioelectricity and Biomagnetism (New York: John Wiley and Sons, 1998).

[4] Darren Hooks, Myocardial segment-specific model generation for simulating the electrical action of the heart, BioMedical Engineering OnLine, 6(1), 2007, 21-21.

[5] Darren A. Hooks, Karl A. Tomlinson, Scott G. Marsden, Ian J. LeGrice, Bruce H. Smaill, Andrew J. Pullan, and Peter J. Hunter, Cardiac microstructure: Implications for electrical propagation and defibrillation in the heart, Circulation Research, 91(4), 8 2002, 331-338.

[6] Darren A. Hooks, Mark L. Trew, Bryan J. Caldwell, Gregory B. Sands, Ian J. LeGrice, and Bruce H. Smaill, Laminar arrangement of ventricular myocytes influences electrical behavior of the heart, Circulation Research, 101(10), 11 2007, e103-112-e103-112.

[7] Bruce Hopenfeld, Jeroen G. Stinstra, and Rob S. MacLeod, The effect of conductivity on st-segment 
epicardial potentials arising from subendocardial ischemia, Annals of Biomedical Engineering, 33(6), 06 2005, 751-763.

[8] Peter R. Johnston, A cylindrical model for studying subendocardial ischaemia in the left ventricle, Mathematical Biosciences, 186(1), 2003, 43-61.

[9] Peter R. Johnston, Approximate solutions for certain bidomain problems in electrocardiography, Physical Review E, 78(4), 2008.

[10] Peter R. Johnston, A finite volume method solution for the bidomain equations and their application to modelling cardiac ischaemia, Computer Methods in Biomechanics and Biomedical Engineering, 13(2), 2010, 157-170.

[11] Peter R. Johnston. A non-dimensional formulation of the passive bidomain equation, Journal of Electrocardiology, 44(2), 2011, 184-188.

[12] Peter R. Johnston, A sensitivity study of conductivity values in the passive bidomain equation, Mathematical Biosciences, 232(2), 2011, 142-150.

[13] Peter R. Johnston and David Kilpatrick, The effect of conductivity values on ST segment shift in subendocardial ischaemia, IEEE Transactions on Biomedical Engineering, 50(2), 2003, 150-158.

[14] Peter R. Johnston, David Kilpatrick, and Chuan Yong $\mathrm{Li}$, The importance of anisotropy in modelling ST segment shift in subendocardial ischaemia, IEEE Transactions on Biomedical Engineering, 48(12), 2001, 1366-1376.

[15] D. Kilpatrick, A. J. Bell, and S. J. Walker, Derived epicardial potentials differentiate ischemic ST depression from ST depression secondary to ST elevation in acute inferior myocardial infarction in man, Journal of the American College of Cardiology, 14, 1989, 695-702.

[16] D. Li, C. Y. Li, A. C. Yong, and D. Kilpatrick, Source of electrocardiographic ST changes in subendocardial ischemia, Circulation Research, 82, 1988, 957-970.

[17] M. C. MacLachlan, J. Sundnes, and G. T. Lines, Simulation of st segment changes during subendocardial ischemia using a realistic 3 -d cardiac geometry, IEEE Transactions on Biomedical Engineering, 52(5), 2005, 799-807.

[18] P. R. Johnston, Cardiac conductivity values - a challenge for experimentalists?, Noninvasive Functional Source Imaging of the Brain and Heart \& 8th International Conference on Bioelectromagnetism (NFSI \& ICBEM), Banff, Canada, 2011, 39-43.
[19] Salil G. Patel and Bradley J. Roth, Approximate solution to the bidomain equations for electrocardiogram problems, Physical Review E (Statistical, Nonlinear, and Soft Matter Physics), 72(5), 2005, 051931.

[20] Mark Potse, Bruno Dubé, and Alain Vinet, Cardiac anisotropy in boundary-element models for the electrocardiogram, Medical \& Biological Engineering \& Computing, 47(7), 2009, 719-729.

[21] D. E. Roberts, L. T. Hersh, and A. M. Scher, Influence of cardiac fiber orientation on wavefront voltage, conduction velocity and tissue resistivity in the dog, Circulation Research, 44, 1979, 701-712.

[22] D. E. Roberts and A. M. Scher, Effects of tissue anisotropy on extracellular potential fields in canine myocardium in situ, Circulation Research, 50, 1982, 342-351.

[23] B. J. Roth, A comparison of two boundary conditions used with the bidomain model of cardiac tissue, $A n$ nals of Biomedical Engineering, 19, 1991, 669-678.

[24] S. Rush, J. A. Abildskov, and R. McFee, Resistivity of body tissues at low frequencies, Circulation Research, 12, 1963, 40-50.

[25] D. D. Streeter, Gross morphology and fiber geometry of the heart, in R. M. Berne (Ed.), Handbook of Physiology, Vol 1, 2 (Baltimore, MD: Williams and Williams, 1979), 61-112.

[26] L. Tung, A Bi-domain model for describing ischaemic myocardial D-C potentials, $\mathrm{PhD}$ thesis, Massachusetts Institute of Technology, June 1978. 